\title{
The Integral of the $n$th Power of the Voigt Function
}

\author{
By Alex Reichel*
}

\begin{abstract}
A series expansion is given for the computation of the integral over $(-\infty, \infty)$ of the $n$th power of the Voigt function for use in spectral line calculations with Doppler broadening. A nine significant figure table is presented for $n$ up to 25 and for a wide range of values of the second parameter on a microfiche card in this issue.
\end{abstract}

Introduction. In studies of the transport of particles or photons in the neighborhood of Doppler-broadened spectral lines, a function of fundamental interest is the Voigt profile

$$
\zeta_{0}(x, t)=\frac{1}{(4 \pi t)^{1 / 2}} \int_{-\infty}^{+\infty} \frac{\exp \left[-(x-y)^{2} / 4 t\right] d y}{1+y^{2}} .
$$

Often, integrals of functionals of $C_{0}(x, t)$ have to be evaluated for a wide range of parameters. As examples we have the "curves of growth"

$$
\begin{aligned}
& K(t, \alpha)=\int_{-\infty}^{+\infty}\left\{1-\exp \left[-\alpha U_{0}(x, t)\right]\right\} d x, \\
& J(t, \beta)=\int_{-\infty}^{+\infty} \frac{U_{0}(x, t)}{U_{0}(x, t)+\beta} d x,
\end{aligned}
$$

and a host of more elaborate integrals which appear as kernels of integral equations. Generally, numerical quadrature is difficult, and often inaccurate due to the occurrence of steep and shifting plateaux in the integrands. Various authors have conceived the possibility of expanding the integrals in series involving integrals of powers of the Voigt function,

$$
\chi_{n}(t)=\int_{-\infty}^{+\infty}\left\{U_{0}(x, t)\right\}^{n} d x
$$

Several attempts have been made to obtain reasonable approximations to $\chi_{n}(t)$, the earliest being that of Shapiro, given by Gurevich and Pomeranchouk [1]. Improvements to this approximation have been given by Keane and McKay [2] and by McKay [3]. A short table of $\chi_{n}(t)$ has been given by Cook and Elliott [4], using numerical quadrature.

This paper shows that $\chi_{n}(t)$ can be computed accurately from a series expansion which is rapidly convergent for values of $t>>1$ and $n>2$, the values of $\chi_{1}(t)$ and $\chi_{2}(t)$ being known. For values of $t<<1$, a range of no interest in fields the author has studied, the series is still convergent but some difficulty may be experienced with cancellation of significant figures. A table is given for values of $n$ up to 25 and for a selection of values of $t$ which reflect the wide parameter range required to cover the various contexts in which the functions occur. This table appears in the micro-

Received August 21, 1968.

* Member of the Joint Institute for Laboratory Astrophysics, Boulder, Colorado (1968); on leave from University of Sydney, Australia. 
fiche section of this issue. Many workers in optics and astrophysics use the Voigt function in the form

$$
\begin{aligned}
H(a, u) & =\frac{a}{\pi} \int_{-\infty}^{+\infty} \frac{\exp \left[-y^{2}\right] d y}{(u-y)^{2}+a^{2}}, \\
& =\frac{1}{a \sqrt{ } \pi} U_{0}\left(\frac{u}{a}, \frac{1}{4 a^{2}}\right)
\end{aligned}
$$

which is normalised to $\sqrt{ } \pi$.

Thus

$$
\int_{-\infty}^{+\infty}\{H(a, u)\}^{n} d u=\frac{1}{a^{n-1} \pi^{n / 2}} \chi_{n}\left(\frac{1}{4 a^{2}}\right)
$$

1. Series Expansion. The function $\chi_{n}(t)$ may be calculated from the formula

$$
\chi_{n}(t)=2(\pi t / n)^{1 / 2}\left[S_{0}{ }^{n}+n \sum_{r=1}^{\infty} A_{r} B_{n, r}\right]
$$

which is derived in Section 2.

Here,

$$
S_{0}=U_{0}(0, t)=\frac{\sqrt{ } \pi}{2 \sqrt{ } t} e^{1 / 4 t} \operatorname{erfc} \frac{1}{2 \sqrt{ } t}=\sum_{n=0}^{\infty} u_{n},
$$

where $u_{0}=\sqrt{ } \pi / 2 \sqrt{ } t, u_{1}=-1 / 2 t, u_{n}=u_{n-2} / 2 t n$. The coefficient $A_{r}$ is given by

$$
A_{r}=\frac{2 r-1}{2 r n} A_{r-1}
$$

with $A_{1}=1 / 2 n$.

Also

$$
B_{n, r}=\sum_{k=0}^{r-1}\left(\begin{array}{c}
r-1 \\
k
\end{array}\right) S_{k} B_{n-1, r-k}
$$

Here,

$$
B_{1, r}=S_{r}
$$

where

$$
S_{r}=\frac{1}{(2 r-1) \cdot 2 t}\left(1-S_{r-1}\right), \quad r=1,2,3, \cdots
$$

2. Derivation. The form

$$
U_{0}(x, t)=\frac{\exp \left[1 / 4 t-x^{2} / 4 t\right]}{\sqrt{ } t} \int_{1 / 2 \sqrt{ } t}^{\infty} \exp \left[\left(x^{2} / 16 t^{2} v^{2}\right)-v^{2}\right] d v
$$

has been given previously by Reichel [5]. Whence,

$$
\chi_{n}(t)=\frac{e^{n / 4 t}}{t^{n / 2}} \int_{1 / 2 \sqrt{ } t}^{\infty} \cdots \int_{1 / 2 \sqrt{ } t}^{\infty} e^{-b} d \mathrm{y}_{i} \int_{-\infty}^{+\infty} \exp \left[-\left(n / 4 t-a / 16 t^{2}\right) x^{2}\right] d x,
$$


where

$$
a=\sum_{1}^{n} 1 / y_{i}^{2} ; \quad b=\sum_{1}^{n} y_{i}^{2}
$$

Thus,

$$
\chi_{n}(t)=\frac{\sqrt{ } \pi e^{n / 4 t}}{t^{n / 2}} \int_{1 / 2 \sqrt{ } t}^{\infty} \ldots \int_{1 / 2 \sqrt{ } t}^{\infty} e^{-b}\left(n / 4 t-a / 16 t^{2}\right)^{-1 / 2} d \mathbf{y}_{i} .
$$

The integral ('.4) converges over the singularity at the corner of the hyperspace.

An expansion in power series gives

$$
\begin{aligned}
\chi_{n}(t)= & \frac{2 \sqrt{ } \pi}{\sqrt{ } n} \frac{e^{n / 4 t}}{t^{(n-1) / 2}} \int_{1 / 2 \sqrt{ } t}^{\infty} \ldots \int_{1 / 2 \sqrt{ } t}^{\infty} \exp \left[-\left(\sum_{1}^{n} y_{i}^{2}\right)\right] d \mathbf{y}_{i} \\
& \times\left\{1+\frac{1}{2}\left(\frac{\sum_{1}^{n} 1 / y_{i}{ }^{2}}{4 t n}\right)+\frac{1 \cdot 3}{2^{2} 2 !}\left(\frac{\sum_{1}^{n} 1 / y_{i}{ }^{2}}{4 t n}\right)^{2}+\cdots\right\} .
\end{aligned}
$$

To evaluate (2.5) term by term we require the multinomial theorem, viz:

$$
\left(a_{1}+a_{2}+\cdots+a_{n}\right)^{R}=\sum_{r, s, t \cdots q} \frac{R !}{r ! s ! t ! \cdots q !} a_{1}^{r} a_{2}{ }^{s} a_{3}{ }^{t} \cdots a_{n}{ }^{q},
$$

where $r+s+t+\cdots+q=R$. The total number of terms of all possible combinations $a_{1}{ }^{r} a_{2}{ }^{s} a_{3}{ }^{t} \cdots a_{n}{ }^{q}$ such that $r+s+t+\cdots+q=R$ is

$$
\left(\begin{array}{c}
R+n-1 \\
n-1
\end{array}\right)
$$

The first term in the series (2.5) is

$$
\begin{aligned}
\int_{1 / 2 \vee t}^{\infty} \cdots \int_{1 / 2 \vee t}^{\infty} \exp \left[-\sum_{1}^{n} y_{i}^{2}\right] d y_{i} & =\left\{\int_{1 / 2 \vee t}^{\infty} \exp \left[-y^{2}\right] d y\right\}^{n}, \\
& =t^{n / 2} e^{-n / 4 t} S_{0}{ }^{n},
\end{aligned}
$$

where $S_{0}=U_{0}(0, t)$.

As a typical example of higher-order terms we consider the fourth term

$$
\frac{1 \cdot 3 \cdot 5}{2^{3} \cdot 3 !(4 t)^{3} n^{3}} \int_{1 / 2 \sqrt{ } t}^{\infty} \ldots \int_{1 / 2 \sqrt{ } t}^{\infty} \exp \left[-\sum_{1}^{n} y_{i}{ }^{2}\right]\left(\sum_{1}^{n} 1 / y_{i}{ }^{2}\right)^{3} d \mathbf{y}_{i} .
$$

In $\left(\sum_{1}^{n} 1 / y_{i}{ }^{2}\right)^{3}$ there are:

$n$ terms of the type $1 / y_{i}{ }^{6}$,

$n(n-1)$ terms of the type $3 / y_{i}{ }^{4} y_{j}{ }^{2}$,

$n(n-1)(n-2) / 3$ ! terms of type $6 / y_{i}^{2} y_{j}{ }^{2} y_{k}{ }^{2}$.

The integral (2.7) then reduces to

$$
\frac{1 \cdot 3 \cdot 5}{2^{3} \cdot 3 ! n^{3}}\left[t^{n / 2} e^{-n / 4 t}\left\{n S_{0}{ }^{n-1} S_{3}+3 n(n-1) S_{0}{ }^{n-2} S_{2} S_{1}+n(n-1)(n-2) S_{0}{ }^{n-3} S_{1}{ }^{3}\right\}\right]
$$

where, in general,

$$
S_{r}=\frac{1}{(2 r-1) \cdot 2 t}\left(1-S_{r-1}\right)
$$


Note that the

$$
S_{r}=\frac{e^{1 / 4 t}}{\sqrt{ } t} \int_{1 / 2 \sqrt{ } t}^{\infty} \frac{\exp \left[-v^{2}\right] d v}{\left(4 t v^{2}\right)^{n}}
$$

are related to the incomplete gamma function. Also, for $t>1 / 2,0<S_{r}<1$, all $r$. Thus $\chi_{n}(t)$ has the rather formidable series expansion:

$$
\begin{aligned}
& \chi_{n}(t)=2(\pi t / n)^{1 / 2}\left[S_{0}{ }^{n}+\frac{1}{2 \cdot 1 !} S_{0}{ }^{n-1} S_{1}+\frac{1 \cdot 3}{2^{3} 2 ! n}\left\{S_{0}{ }^{n-1} S_{2}+(n-1){S_{0}}^{n-2}{S_{1}}^{2}\right\}\right.
\end{aligned}
$$

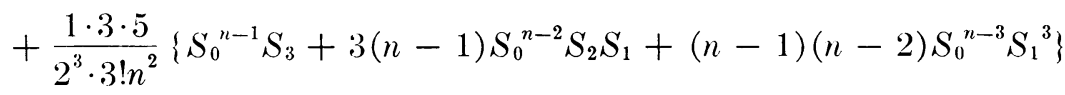

$$
\begin{aligned}
& +\frac{1 \cdot 3 \cdot 5 \cdot 7}{2^{4} \cdot 4 ! n^{3}}\left\{S_{0}^{n-1} S_{4}+4(n-1) S_{0}^{n-2} S_{3} S_{1}+3(n-1) S_{0}^{n-2}{S_{2}}^{2}\right. \\
& \left.+6(n-1)(n-2) S_{0}{ }^{n-3} S_{2} S_{1}{ }^{2}+(n-1)(n-2)(n-3) S_{0}{ }^{n-4} S_{1}{ }^{4}\right\} \\
& +\frac{1 \cdot 3 \cdot 5 \cdot 7 \cdot 9}{2^{5} \cdot 5 ! n^{4}}\left\{S_{0}{ }^{n-1} S_{5}+5(n-1) S_{0}{ }^{n-2} S_{4} S_{1}+10(n-1) S_{0}{ }^{n-2} S_{3} S_{2}\right. \\
& +10(n-1)(n-2) S_{0}{ }^{n-3} S_{3} S_{1}{ }^{2}+1 \tilde{j}(n-1)(n-2) S_{0}{ }^{n-3} S_{2}{ }^{2} S_{1} \\
& +10(n-1)(n-2)(n-3) S_{0}{ }^{n-4} S_{2} S_{1}{ }^{3} \\
& \left.\left.+(n-1)(n-2)(n-3)(n-4) S_{0}^{n-5} S_{1}^{{ }^{5}}\right\}+\cdots \cdots\right] \text {. }
\end{aligned}
$$

Further terms are readily obtained. By writing down the series for $n=1,2,3, \cdots$ and comparing terms, the recurrence relation (1.4) is easily established and Eq. (1.1) follows.

Values of $\chi_{n}(t)$ for $n=1,2$ can be written down exactly:

$$
\begin{aligned}
& \chi_{1}(t)=\int_{-\infty}^{+\infty} C_{0}(x, t) d x=\pi, \\
& \chi_{2}(t)=\int_{-\infty}^{+\infty} C_{0}^{2}(x, t) d x=\frac{\pi}{2} L_{0}(0, t / 2) .
\end{aligned}
$$

The second of these two formulae provides a useful check on the series (1.1) whose convergence is slowest for the smallest values of $n$ and $t$. Details of the computation are given in Section 3.

3. Computation. The computation was designed for an overall accuracy of more than nine significant figures by terminating the series after the term less than $\left(A_{1} B_{n, 1}\right) \times 10^{-12}$. The order of the binomial matrix required in Eq. (1.4) is equal to the number of terms of the series necessary to give the required accuracy. This matrix is readily generated, using the Pascal triangle, after first generating a square matrix of zeros.

For $n=2$, the series is slowly convergent, requiring over 200 terms. These 200 terms give nine significant figure accuracy only when $t>10^{4}$, and only four significant figure accuracy from $t \geqq 1$. Accordingly, the values for $n=2$ are computed from Eq. (2.10) using the subroutine (1.2). It is convenient to compute $\chi_{2}(t)$ after 
the computation for higher values of $n$ is complete, since the series coefficients for $n=2$ are required in any case.

The next most difficult case, for $n=3$ and $t=1$, requires just under 200 terms. This fixes the dimensions of the binomial matrix used in the calculations. The number of terms can be dramatically reduced if less accuracy is specified or if working with higher values of $t$. For $n>2$ and $t>10$, eight significant figures are obtained in less than 100 terms, and six significant figures in less than 50 terms.

For $n>25$, the first six terms of the series, Eq. (2.8), may be used with the following accuracy specifications: for

$$
\begin{aligned}
1<t<10, & \text { four figures, } \\
10<t<25, & \text { five figures, } \\
25<t & <100, \text { six figures, } \\
100<t<150, & \text { seven figures, } \\
150<t<1000, \text { eight figures, } & \\
t & >1000, \text { nine figures. }
\end{aligned}
$$

The main programme may still be used for $n>25$ if machine space is available. If one is only interested in values of $n>25$, the binomial matrix need only be (30,30) say for all $t \geqq 1 ;(15,15)$ say for $t>50$, and $(10,10)$ say for all $t>1000$. The computation must still commence at $n=2$.

Joint Institute for Laboratory Astrophysics

Boulder, Colorado 80302

1. I. I. Gurevich \& I. Y. Pomeranchouk, Proc. First Internat. Conf. Peaceful Uses Atomic Energy, (Geneva), v. 5, 195.), p. 466, p. 649 .

2. A. Keank \& M. H. MCKAY, "An approximation arising theory of resonance absorption," Austral. J. Appl. Sci., v. 11, 1960, p. 321.

3. M. H. MCKAY, "An improvement on Shapiro's approximation to a function occurring in the theory of resonance absorption," J. Nuclear Sci. Tech., v. 2, 4, 1965, p. 117.

4. J. L. COOK \& I). ELLIOTT, "The tabulation of three functions arising in nuclear resonance theory," Austral. J. Appl. Sci., v. 11, 1960, pp. 16-32. MR $22 \# 6130$.

5 . A. Reicheis, "Doppler broadening integrals and other relatives of the error function," J. Quant. Spect. Radiat. Transfer, v. 8, 1968, p. 1601. 